\title{
An environment adaptive evaluation method based on the effect for electronic equipment in southeast coastal area
}

\author{
ZHOU Xiao-lan, LIU Shi-jun \\ Army Artillery Air Defense Academy, Hefei, 230031, China
}

Key words: environmental factors; environmental worthiness; grey correlation analysis; influence

\begin{abstract}
Analysis of the environmental factors in the southeast coastal area influence the efficiency of electronic equipment, establish the key indicators of environmental adaptability. The grey correlation analysis method is introduced into the electronic equipment environmental worthiness evaluation, combined with the fuzzy mathematics and expert scoring method, through the analysis of association degree of environmental impact factors and adaptive, identification of effect of environmental factors on adaptability. Through the calculation of a certain type of electronic equipment adaptability model in the southeast coastal region, show that it is feasible and effective.
\end{abstract}

\section{基于效果的东南濒海地区电子装备环境适应性评价方法}

\author{
周晓兰 刘士军 \\ (陆军炮兵防空兵学院 合肥 230031)
}

关键词: 环境因素, 环境适应性, 灰色关联分析, 影响效果

摘 要: 分析东南濒海地区影响电子装备效能的主要环境因素, 建立环境适应性的关键指标, 将灰色关联度分析引入到电子装备环境适应性评估中, 结合模糊数学及专家评分法, 分析环 境影响因素与适应性指标的关联度, 找出环境因素对装备适应性指标的影响效果。通过对东 南濒海地区某型号电子装备适应性模型的计算，表明了方法的可行性和有效性。

\section{1 引言}

环境适应性是电子装备重要的质量特性，这个特性对于装备效能的发挥具有至关重要的 作用。恶劣的自然环境和电磁环境对电子装备的性能会产生显著的影响, 特别是对使用可靠 性和人一机环境等产生直接影响, 甚至会导致电子装备的效能明显降低。东南沿海地区战场环 境特殊性显著, 也是电子装备使用的主要地域之一。基于环境因素对电子装备实际影响效果, 采用指标化定量化评价方法分析该地域环境下电子装备环境适应性, 对于电子装备使用与保 障具有重要价值。

\section{2 东南濒海地区环境对陆战电子装备的影响效果分析}

综合分析自然环境和电磁环境对电子装备的影响效果主要分为以下几个方面: 元件损坏 导致装备系统故障, 元件敏感度降低导致装备响应度低, 器件失效导致装备效能减弱, 以及装 备整体寿命缩短等。 
表1 东南濒海地区环境适应性影响因素及故障表现

\begin{tabular}{|l|c|c|c|}
\hline \multicolumn{2}{|c|}{ 主要环境因素 } & 影响方式 & 故障表现 \\
\hline 1 & 高 温 & 老化软化 & 电机、变压器、电容器等器件较易被击穿 \\
\hline 2 & 湿 气 & 锈蚀腐蚀 & 击穿、短路、漏电、打火等 \\
\hline 3 & 盐 雾 & 材料腐蚀 & 绝缘材料腐蚀, 影响电子性能 \\
\hline 4 & 机械冲击 & 机械应力 & $\begin{array}{c}\text { 结构失效, 集成电路模块元件管脚、引线折断, 电子接插件性 } \\
\text { 能下降, 输出脉冲超过预订要求, 信号异常 }\end{array}$ \\
\hline 5 & 雷 电 & 瞬间高压 & 高压击穿 \\
\hline 6 & 电磁干扰 & 电磁效应 & $\begin{array}{l}\text { 器件烧毁, 电涌冲击, 瞬时干扰, 微波加热, 强电场效应, 设 } \\
\text { 备电子器件的永久性失效, 甚至烧毁内部半导体器件 }\end{array}$ \\
\hline
\end{tabular}

\section{3 东南濒海地区电子装备环境适应性评价体系}

电子装备对特定环境的适应性，就是电子装备在其全寿命管理期内，在该环境可能遇到 的各种条件下, 达到其战术技术性能要求, 并完成作战任务的能力。为了衡量电子装备对环 境的适应性, 应采用某种定量尺度去度量一定条件的恶劣环境因素下装备的实际效能。电子 装备环境适应性评估的重要内容之一就是确定复杂环境适应性指标, 由于环境适应性指标的 特殊性, 使得其评估指标不能像战术技术指标那样可以直接度量, 但是适应性指标必须反映 该装备在复杂环境下的作战效能。选定东南濒海地区电子装备环境适应性评价指标：其一, 要重点反映环境效应特征, 实现系统性评价的目标; 其二, 评价要素和层次应尽可能结构化, 便于分析和计算；其三，具体问题具体分析，具有充分的灵活性。

基于效果的电子装备环境适应性评价实质就是，在东南濒海地区各类因素影响下，评估 电子装备实现所有预定功能与性能受影响的程度。电子装备在此特殊环境中必须具有 “战备 完好性” 和 “持续作战能力” ，装备的 “战备完好性” 可以由装备的可用性、使用可信度来 度量, 装备的 “持续作战能力” 可以由装备的使用可信度、保障性来度量。基于这两个方面 的考虑, 建立环境适应性的指标体系如下:

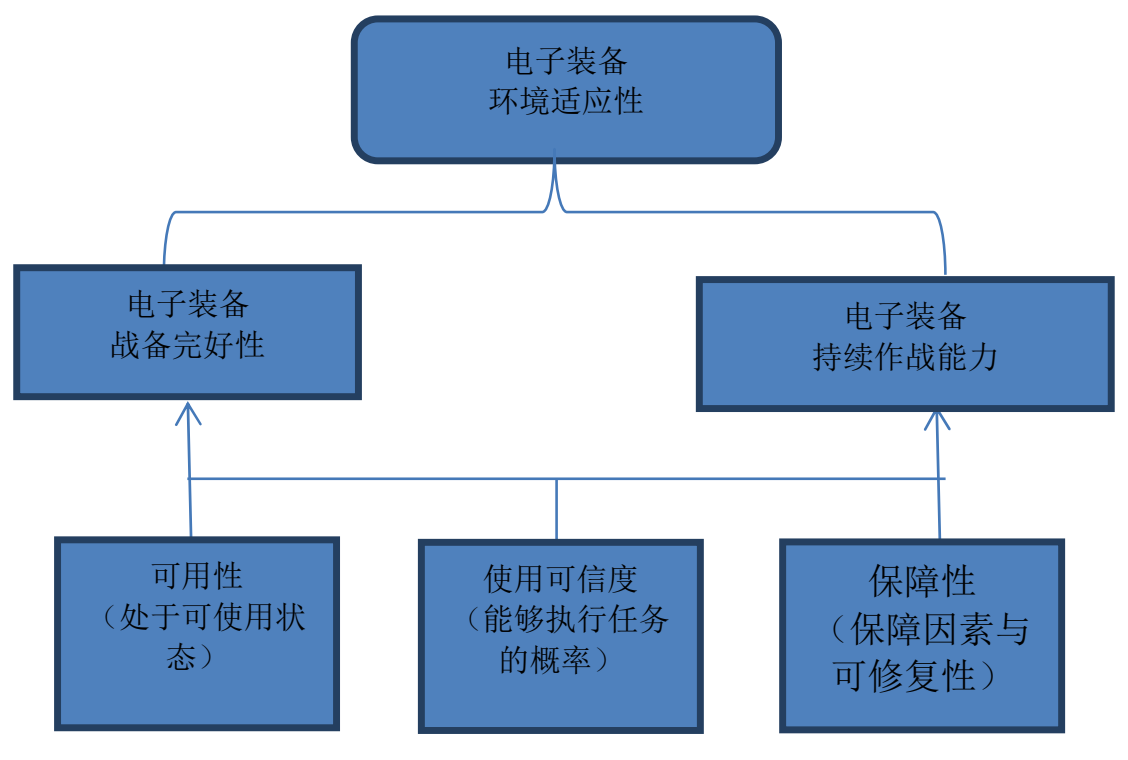

图1 电子装备环境适应性评价体系

图1中，电子装备的环境适应性由 “战备完好性” 和 “持续作战能力” 表征。指标 “战备 完好性” 是在一定的环境条件下, 电子装备及时投入部署和使用, 并且发挥作用的能力; “持 续作战能力” 则是指在一定环境条件下, 电子装备按照必要的强度和持续时间执行作战任务, 以达到完成作战任务的能力。 
电子装备的 “战备完好性”，由装备的可用性、使用可信度来度量。可用性是指装备处 于可使用状态; 可用性是可靠性和维修性的综合体现。使用可信度是装备在需要时能够执行 其规定任务的概率。“持续作战能力” 由装备的使用可信度和保障性来度量。保障性是指电 子装备在一定环境下、在规定的任务持续时间内, 维持该装备正常工作所需的各类保障因素， 这里主要是装备利用率问题。

\section{4 基于灰色理论建立电子装备环境适应性模型}

\section{1 电子装备环境适应性影响因素的数值分析}

在分析东南濒海地区对电子装备环境适应性的影响因素时，可采取数值转换方法，以高 温为例, 可分为 “超高温、高温、较高温度、一般高温、适宜温度” 五个等级, 对应数值为 “1、3、5、7、9” ，相对应的气温为 “ $40^{\circ} \mathrm{C}$ 以上、 $36^{\circ} \mathrm{C}-40^{\circ} \mathrm{C} 、 32^{\circ} \mathrm{C}-36^{\circ} \mathrm{C} 、 28^{\circ} \mathrm{C}-32^{\circ} \mathrm{C} 、 28^{\circ} \mathrm{C}$ 以下”，再进一步考察一年当中该地区各种气温条件的小时数。分析湿气，盐雾、机械冲击、 雷电、电磁干扰时, 也需要建立相应的分级, 并考察该因素各个等级内的数值, 得到该地区 的环境数值分析模型。

\section{2 基于模糊数学的评价尺度确立}

评价电子装备对于环境的适应性首先是以定性语言表现出来的, 如某装备在高温环境下 可用性是 “良好” , 对该类评价语言进行量化, 不能采用一般数值, 可以采用模糊数或模糊 子集。

基于模糊数学的数值量化方法的关键就是确定性能指标的极值参考标准和构造数据语言 标度的二元对比矩阵, 也就是确立评价尺度。评价尺度是对各层次评价指标的一种语言描述, 它是评审人员对各评价指标所给出的评价集合, 本文中评价尺度按照电子装备环境适应性的 问题背景, 选择 5 个语气算子, 构成 4 个语气算子级差, 将模糊语气算子和模糊标度形成对照 的评价尺度对照表。

表2 评价尺度对照表

\begin{tabular}{|c|c|c|c|c|c|}
\hline 语气算子 & 低 & 较低 & 良好 & 较高 & 高 \\
\hline 模糊标度 & 1 & 3 & 5 & 7 & 9 \\
\hline
\end{tabular}

\section{3 专家打分法确定适应性指标隶属度}

考察某种电子装备的适应性指标隶属度可以通过专家综合评议的方法, 来确定隶属度矩 阵 $V=[v 1, v 2, v 3, v 4, v 5]$ 。首先确定评语集, 见表二。然后进行专家评语。专家评语是指请一 批相关领域的专家对指标打分。打分时并不要求给出具体数值, 而是在 5 个评语级别 “低、较 低、良好、较高、高” 上选择最合适的级别即可。打分时给出相关资料, 请各位专家予以参 阅，力求打分客观公正。最后，将各位专家对各指标的评分表进行汇总。

\section{4 基于灰色关联分析法的环境适应模型}

灰色关联分析的基本思想是根据序列曲线几何形状的相似程度来判断其联系是否紧密。 该方法从定量角度对统计数据序列中的系统特征序列 (参考数列) 和相关因素序列 (比较数列) 进行量化, 求解两者之间的关联度, 根据关联度数值的大小来体现系统特征序列与相关因素 序列的关联程度。灰色关联分析的思路是先初始化原始数据序列, 然后计算关联系数得到关 联矩阵及关联度, 最后根据关联度数值的大小对相关因素序列进行排序。

设系统特征行为序列

$$
\begin{aligned}
& X_{0}=\left(x_{0}(1), x_{0}(2), \cdots, x_{0}(n)\right) \\
& X_{1}=\left(x_{1}(1), x_{1}(2), \cdots, x_{1}(n)\right)
\end{aligned}
$$




$$
\begin{gathered}
X_{i}=\left(x_{i}(1), x_{i}(2), \cdots, x_{i}(n)\right) \\
\cdots \cdots \\
X_{n}=\left(x_{n}(1), x_{n}(2), \cdots, x_{n}(n)\right) \\
\text { 对于 } \rho \in(0,1) \text {, 令 } \\
\xi\left(x_{0}(k), x_{i}(k)\right)=\frac{\min _{i} \min _{k}\left|x_{0}(k)-x_{i}(k)\right|+\rho \max _{i} \max _{k}\left|x_{0}(k)-x_{i}(k)\right|}{\left|x_{0}(k)-x_{i}(k)\right|+\rho \max _{i} \max _{k}\left|x_{0}(k)-x_{i}(k)\right|}
\end{gathered}
$$

则 $\xi\left(\mathrm{X}_{0}, X_{i}\right)$ 称为 $\mathrm{X}_{0}$ 与 $\mathrm{X}_{\mathrm{i}}$ 的灰色关联度 (Grey Correlation Degree), $\xi\left(x_{0}(k), x_{i}(k)\right)$ 为 $\mathrm{k}$ 点关联系数, 记为 $\xi_{0 \mathrm{i}}(\mathrm{k})$ 。这也就是本文所采用的模型。

在求解灰色关联度的过程中, 有以下几个步骤:

（1）考虑到各数据列的单位或初值不同，为使其具有可比性，需对原始数据进行无量纲 化处理, 以便比较不同量纲或不同量级的数据, 常用的是数据的初值化或均值化, 此处采用 初值化。

（2）求差序列，差序列的表达式如下:

$$
\Delta_{i}(k)=\left|x_{0}^{\prime}(k)-x_{i}^{\prime}(k)\right|, \Delta_{i}=\left(\Delta_{i}(1), \Delta_{i}(2), \cdots, \Delta_{i}(n)\right), \quad i=1,2, \cdots, m
$$

(3) 求两极最大差与最小差

$$
M_{1}=\max _{i} \max _{k} \Delta_{i}(k), M_{2}=\min _{i} \min _{k} \Delta_{i}(k)
$$

(4) 求关联系数。关联系数是依据因素数据列的几何形状、发展趋势的接近情况来衡量 关联系数的依据, 即

$$
\xi_{0 i}(k)=\frac{\mathrm{M}_{2}+\rho \mathrm{M}_{1}}{\Delta_{\mathrm{i}}(k)+\rho \mathrm{M}_{1}}, \xi \in(0,1), k=1,2, \ldots, \quad \mathrm{m}
$$

其中, $\rho$ 为分辨系数, $\rho$ 越小, 分辨能力越大。当 $\rho$ 满足 $\rho \leq 0.5463$ 时, 达到最好的分辨能 力, 一般取值 $\rho=0.5$ 。

（5）计算关联度。因为关联序列中数据很多，信息不够集中，比较不便，所以有必要 把各个不同时刻的关联系数归纳为一个具体值。求关联系数列的平均值就是将这种信息集中 处理的一种方法。这个平均值就是关联度。

$$
\gamma_{0 i}=\frac{1}{n} \sum_{i=1}^{n} \xi_{0 i}(k), i=1,2, \ldots \mathrm{m}
$$

\section{5 东南濒海地区电子装备环境适应性模型检验}

我国东南沿海地区具有自然环境高温、潮湿、雨多、盐雾重等特点, 电子装备需要加强 防潮、防热、防霉烂工作。另外, 由于电子战和信息战成为现代战争的主要样式, 在数十到 数百平方公里的战场上，部署着数以千计的各种普戒、搜索、跟踪、制导等敌我双方雷达，发 射功率从几百瓦到几千千瓦, 频率从 I 波段直至毫米波段, 再加上通讯、人为和自然电磁干 扰等，从而构成了异常复杂的电磁环境。

因此，在具体检查某电子装备的环境适应性时，要查阅当地的自然条件和电子作战条件。 以福建省某近海岛屿为例, 此岛屿为典型的亚热带气候, 受海洋季风影响较大, 同时也 
是处于台海最前沿。此环境比较特殊, 结合当地的观察数据可得到该环境下的各项适应性影 响因素的评价指标值。再进行均值化处理有以下结果:

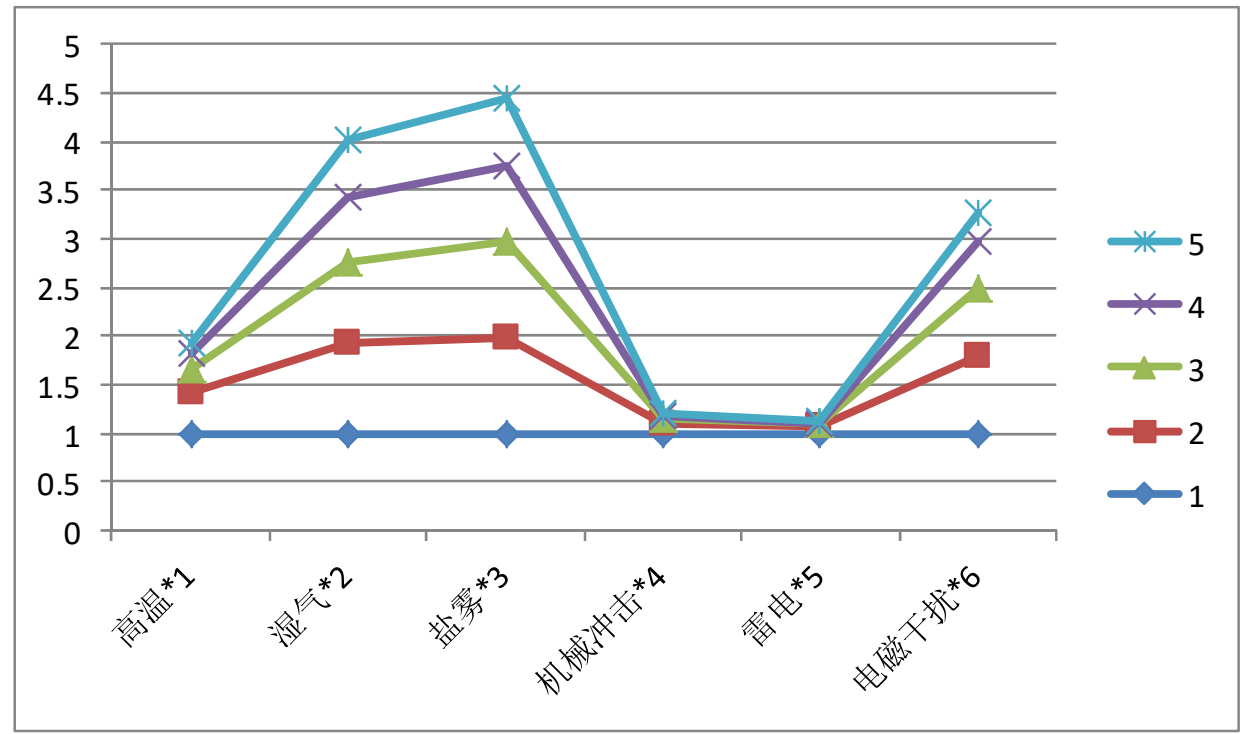

图2 均值化处理后的影响因素

专家对某型号装备三个环境适应性指标评审之后的结果，也可以进行相应的均值化处理， 并得到以下结果:

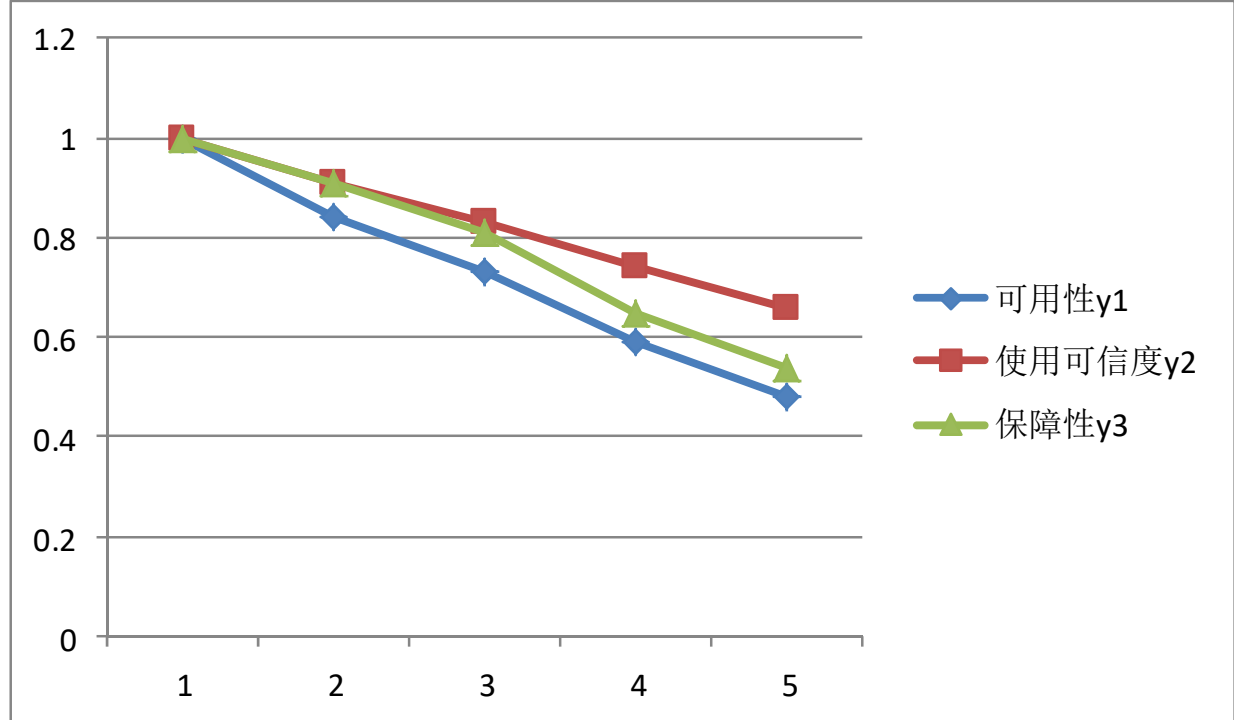

图3 均值化处理后的专家评审结果

通过关联度的计算式可分别计算环境适应性模型中，可用性、使用可信度、保障性与各 影响因素的关联，见下表:

表3 适应性指标与各影响因素的关联度

\begin{tabular}{|c|c|c|c|c|c|c|}
\hline 5 & $\mathrm{x} 1$ & $\mathrm{x} 2$ & $\mathrm{x} 3$ & $\mathrm{x} 4$ & $\mathrm{x} 5$ & $\mathrm{x} 6$ \\
\hline $\mathrm{y} 1$ & 0.64 & 0.78 & 0.67 & 0.59 & 0.58 & 0.79 \\
\hline $\mathrm{y} 2$ & 0.59 & 0.79 & 0.84 & 0.58 & 0.60 & 0.66 \\
\hline $\mathrm{y} 3$ & 0.55 & 0.95 & 0.79 & 0.49 & 0.49 & 0.76 \\
\hline
\end{tabular}

不同因素与同一指标之间的关联度顺序为: 


$$
\frac{1}{6} \sum_{i=1}^{6} \xi_{i 2}=0.6766>\frac{1}{6} \sum_{i=1}^{6} \xi_{i 1}=0.6750>\frac{1}{6} \sum_{i=1}^{6} \xi_{i 3}=0.6717
$$

即 $\gamma_{02}>\gamma_{01}>\gamma_{03}$

可知 $\mathrm{y} 2>\mathrm{y} 1>\mathrm{y} 3$, 即按照排序, 依次对应为使用可信度、可用性和保障性, 说明各种环境因 素与装备的使用可信度关联度最大, 即该型号装备的使用可信度受各种环境因素的影响最大。

\section{6 总结}

严酷的自然环境和复杂的电磁环境会严重影响和制约电子装备的效能发挥和质量可靠 性。电子装备的环境适应性评价研究还处在发展的过程中, 本文所提出的基于效果的适应性 评价方法主要是从电子装备作战效能发挥最重要的几个指标出发, 采用灰色关联度方法, 探 索各项环境因素的影响效果, 最终得到电子装备的环境适应性指标与环境因素的关联度。该 方法可以用于研究装备各个环境适应指标的排序, 也可用于环境适应性这一最终指标的研究。 在下一步的研究中, 需要深入调查, 结合装备的具体实际, 对各项参数进行论证和修改, 从 而对电子装备环境适应性研究有促进意义。

\section{参考文献:}

[1] 王群, 陈之光, 汪文峰. 基于灰色理论的电子装备环境适应性评判模型 $[\mathrm{J}]$, 装备环境工程, 2010.12(6), 94-98

[2] 柯宏发等.电子装备复杂电磁环境适应性试验与评估[M], 北京：国防工业出版社, 2015, 136-146

[3] 赵红言, 张君, 张建强. 灰色关联分析法在电子设备故障树中的应用 [J], 实验室研究与探索, 2014.3 (3) , 21-24 